\title{
Eyelid Surgery in Dysthyroid Ophthalmopathy
}

\author{
A HEDIN \\ Stockholm, Sweden
}

\begin{abstract}
Summary
Dysthyroid ophthalmopathy often gives rise to functional and cosmetic problems due to eyelid retraction. There are several causes of this state which are usually most obvious in the upper lid. Surgery should be delayed until the condition is stable and other operations have been done. A number of operative procedures have been used. In the upper eyelid, the traction of the Müller muscle and, most often, the levator is released; in the lower eyelid, the retractors are recessed or excised. Spacers have been used especially in the lower lid.

The retractors can be reached either through the skin or the conjunctiva. Both methods give good results. In the upper lid, the risk of damaging other structures is lower with a skin incision; in the lower lid the conjunctival incision gives easier access to the tissues. The complications most frequently seen are over- or undercorrection both of which may necessitate a reoperation.
\end{abstract}

In dysthyroid ophthalmopathy, there are cosmetic and functional eyelid problems related to the increased volume and altered characteristics of the orbital tissues and eyelid muscles. Bulging of the orbital tissues often gives rise to a cosmetically annoying fullness of the eyelids, which can be cured by blepharoplasty. Surgically more demanding is correction of upper and/or lower eyelid retraction, conditions which give rise to a 'staring' look and often to considerable discomfort.

\section{Causes of eyelid retraction}

A number of factors have been listed in the explanation of eyelid retraction (Tables I and II $\left.{ }^{1-6}\right)$. Müller muscle overaction certainly plays a role, because an improvement often can be accomplished by sympatholytic agents. These drugs (e.g. guanethidine), although effective, are not suited for long-term treatment because of their locally irritating sideeffects. With increasing proptosis, the eyelids ride up on the globe. Retraction can, how-
Table I Possible causes of upper eyelid retraction

Increased sympathetic tone--overaction of the Müller muscle

Proptosis

Contracture and fibrosis of the inferior rectus muscle, increased tone of the superior rectus and levator muscles

Levator muscle contracture/fibrosis/atrophy

Abnormal adhesions between levator muscle and overlying tissues

Overmedication

Table II Possible causes of lower eyelid retraction

Increased sympathetic tone-overaction of the Müller muscle

Proptosis

Contracture of the superior rectus muscle, increased tone of the inferior rectus muscle

Recession of the inferior rectus muscle

Adhesions and fibrosis

Overmedication

ever, also be prominent in cases of normal eyeball position, and there is no correlation 
between the degree of proptosis and that of retraction. ${ }^{1}$ The inferior rectus muscle is the extraocular muscle that most often becomes fibrotic in orbital disease. This being the case, the superior rectus muscle and the synergistic levator are put under increased stress causing the upper lid to be raised. The levator muscle itself is also involved in orbital inflammation. Microscopy of specimens taken at the time of corrective surgery, i.e. later in the disease, has shown fat infiltration, collagen proliferation and muscle atrophy. ${ }^{3}$ Clinically, contracture of the levator muscle is shown by resistance on downward traction ('Grove's sign'). At surgery, adhesions between the levator aponeurosis and structures on the superior and inferior side are often found. ${ }^{2.3}$ In the lower eyelid, retraction is common after recession of the inferior rectus muscle; the muscle pulls the lid downwards by traction via the inferior aponeurosis.

\section{Indications for surgery}

Patients are always cosmetically disturbed by the staring look. Many conceal their eyes behind dark sunglasses or avoid situations that involve close contact with other people. The widened eyelid fissure makes possible an increased evaporation with drying of the ocular surfaces, resulting in burning, tearing etc. Many patients complain of particular problems in windy conditions. Insufficient closure at night is common with severe ocular discomfort in the morning. Keratitis due to eyelid closure difficulties is, fortunately, not very frequent.

Any of these problems constitutes an indication for surgery which, properly performed, gives significant relief.

\section{Timing of surgery}

If the condition demands a decompression, this should be performed first since it may influence the degree of retraction. Any surgery on the extraocular muscles should also be finished for the same reason (Fig. 1). It is usually recommended that the condition be stable for at least 6 to 12 months. Only in the case of a threatening keratitis can surgery be considered in the active disease state. In such cases, reoperation may be necessary later.

\section{Operative procedures}

First, it should be remembered that in mild cases a lateral tarsorraphy can give a satisfactory result. By this means the eyelid fissure is reduced where it is usually widest, i.e. laterally; the operation corrects the position of both the upper and the lower lid.

In order to relieve moderate or severe retraction, it is necessary to lengthen the structures that pull the lid upwards or downwards respectively. The aim is to obtain a normal eyelid position with good movements and a satisfactory cosmesis. During the operation, nearby tissues like the lacrimal apparatus must not be damaged. The goals can be reached in various ways and a number of surgical procedures have been described. ${ }^{1-15}$ Some are more easily performed than others or are better suited to a certain objective. Tables III and IV list the procedures.

Upper eyelid

The conjunctiva can be divided and recessed

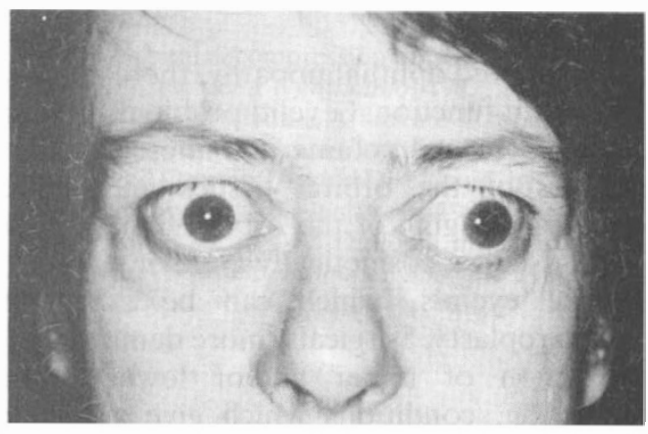

a

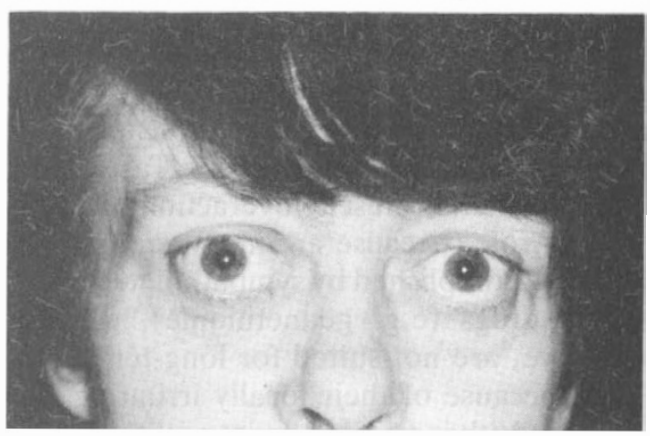

b

Fig. 1. Patient ( $a$ ) before and ( $b$ ) after operation. In this case, squint surgery was performed before correction of the upper eyelid retraction. The patient did not want correction of the lower eyelid retraction. 
Table III Methods of upper eyelid correction

\begin{tabular}{l} 
Conjunctiva \\
Division \\
Müller muscle \\
Division at tarsal base \\
Excision \\
Recession \\
Levator muscle \\
Partial or complete disinsertion from tarsus \\
Partial or complete division \\
Insertion of spacer \\
$\quad$ Sclera \\
$\quad$ Cartilage \\
Tarsal flaps \\
Stripping \\
Recession with fixation to \\
orbicularis and skin \\
anterior tarsal surface \\
conjunctiva \\
Division of adhesions \\
Reverse Frost suture \\
\hline
\end{tabular}

with the Müller muscle and the defect left to re-epithelialise. ${ }^{13}$ Transconjunctival procedures necessitate dividing the conjunctiva; in these procedures it is usually reattached to the tarsal base at the end of the operation. Delicate surgical handling reduces the risk of harm to the conjunctival glands. In longstanding severe cases, subconjunctival fibrosis may itself cause retraction and if so calls for a careful dissection.

The Müller muscle is sometimes found to be thin and fleshy and sometimes thick and fibrotic. In the case of contracture and fibrosis, the muscle must be excised. This is often done in other cases too, because the Müller muscle does not work normally and the excision eliminates overaction. Since the retraction is usually most obvious laterally, it is essential that the lateral $\frac{2}{3}-\frac{3}{4}$ be excised/recessed.

Table IV Methods of lower eyelid correction

\begin{tabular}{l}
\hline Conjunctiva \\
Division \\
Müller muscle and inferior aponeurosis \\
Division \\
Excision \\
Insertion of spacer \\
Sclera \\
Cartilage \\
Tarsal flaps \\
Correction of lower eyelid laxity \\
Lateral canthal tightening \\
Fascia lata sling
\end{tabular}

In the various procedures used, the Müller muscle has been divided at the tarsal base and left to retract, it has been excised or it has been recessed and resutured.

In four recent cases of transconjunctival corrections, I recessed the muscle about $10 \mathrm{~mm}$ instead of doing the standard excision. At re-examination, these four patients did not differ from those that had an excision and there was no measureable change in eyelid position after local sympathomimetic stimulation. This being the case and considering that an excision is more easily performed, my view is that a Müller muscle excision is preferable.

In mild cases, excision/recession of the Müller muscle is sufficient. ${ }^{6.7 .13 .15 .16}$ However, one often has to add a recession of the levator muscle. First, the desired lengthening has to be achieved and secondly, it has to be kept stable. The aponeurosis can be simply disinserted from the tarsus ${ }^{13}$ or it can be divided anywhere along its course including marginal myotomies. ${ }^{3}$ A complete division easily gives rise to a flat eyelid contour, and it is desirable to keep an attachment in the centre of the lid. This can be done by overlapping tenotomies or by partial piece-by-piece stripping. ${ }^{15}$ It is particularly important that the horns of the aponeurosis be cut, since remaining retraction, especially laterally results. During the operation, which is preferably done under local anaesthesia, the eyelid position is repeatedly checked until the desired level is reached.

There are various opinions as to the best way of keeping the eyelid at that level. Some surgeons leave the tissues to heal,${ }^{6.13 .15}$ while others suture the levator at the recessed position. ${ }^{2.5 .7}$ In my experience of 85 eyelids, no suturing has been necessary, neither have I found it desirable to interpose a spacer between the tarsus and the retractors. ${ }^{9.10 .11 .12}$ Many surgeons routinely keep the eyelid down with a reverse Frost suture in the immediate postoperative phase; I use it only in cases of supposed undercorrection.

In severe cases, a proper eyelid position is obtained first when the levator aponeurosis has been freed from adhesions to the orbicularis muscle.

All structures involved can be reached 
either via the skin or through the conjunctiva. Each has its advantages (Table V) and excellent results have been obtained with both approaches. ${ }^{5,11.16}$ Although it is easier to divide the levator horns the cutaneous way, it can also be done from the inside of the eyelid. Dissection of the conjunctiva should be careful laterally so that the lacrimal ductules are not severed. An abnormal position of the lid crease is common after a levator recession, the attachments to the orbicularis are naturally moved upwards. This cosmetic blemish is avoidable if the operation is performed with a skin incision.

In 1985, I reviewed the results of 41 upper eyelids operated on by the conjunctival route. ${ }^{17}$ Since then, another 44 lids have been operated on with a similar procedure and the results represent the experiences of the last two years. The 27 patients $(22$ female and 5 male) were between 28 and 74 years. In all cases the condition had been stable for more than 6 months. The indication was cosmetic in all patients and in 22 eyes there were also functional problems with dryness etc. The degree of retraction varied and details are given in Table VI.

Müllerectomy alone was not sufficient in any case. In 40 eyelids, excision of the Müller muscle was combined with a division of the levator aponeurosis. In 18 eyelids, three overlapping tenotomies were done leaving an

Table V Advantages of cutaneous approach to upper eyelid retractors

Lower risk of damage to the lacrimal ducts

Simultaneous excision of excess skin and fat possible

No sutures to rub the eye

Lid crease can be placed correctly

Better access to levator aponeurosis adhesions

Advantages with the conjunctival approach to the upper eyelid retractors

No visible scar

Easier preparation of the Müller muscle

Table VI Preoperative degree of retraction in primary gaze measured from $1 \mathrm{~mm}$ below limbus (number of eyes)

\begin{tabular}{lrr}
\hline Mild & $1-2 \mathrm{~mm}$ & 3 \\
Moderate & $3-4 \mathrm{~mm}$ & 33 \\
Severe & $>4 \mathrm{~mm}$ & 8 \\
\hline
\end{tabular}

Table VII Functional and cosmetic postoperative condition of 40 eyelids re-examined (number of eyes)

Excellent 24

Good $\quad 12$

Good after reoperation (overcorrected) 2

Overcorrected without further surgery 2

attachment in the central part of the lid. In the other 22 eyelids, several tenotomies or an almost complete division of the aponeurosis was done. In four eyelids, the Müller muscle was recessed and thereafter three tenotomies cut in the aponeurosis. In several cases, additional division of adhesions was performed; a reverse Frost suture was used in three eyes.

The results are shown in Table VII (Fig. 2). Most patients were very satisfied with the lowering of the upper eyelid. Those who had a raised position of the lid crease did not want any corrective surgery; in only two cases was there an indication for a subsequent operation on the lower eyelid.

Not one of 81 eyes re-examined after a transconjunctival operation has shown a dry
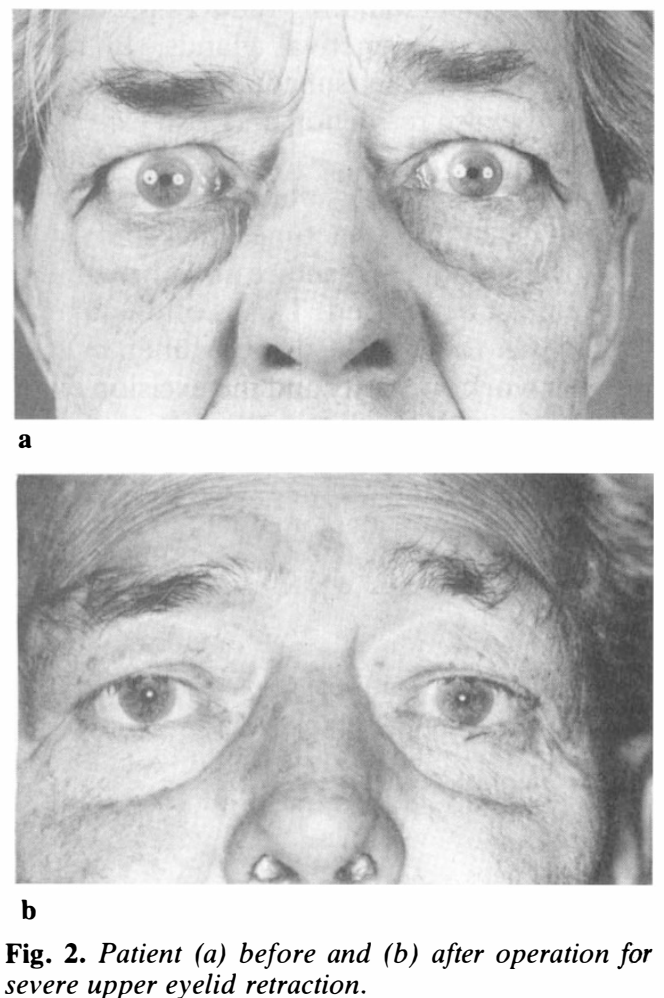
eye syndrome. In 10 eyes of the later series a Schirmer test was done; the wetting was within normal limits in all cases.

\section{Lower eyelid (Table IV)}

In the upper eyelid, the operation is supported by the orbicularis action and by gravity. In the lower eyelid, gravity counteracts the surgery performed so that additional measures often become necessary.

The conjunctiva may be divided and left retracted. Usually it is severed from the tarsal base in order to allow access to lower eyelid retractors. In the lower eyelid, the tiny Müller muscle, the inferior aponeurosis and the orbital septum all join before attaching to the tarsal base. ${ }^{18}$ The retractors must be divided or excised; it is often also necessary to sever the septum. Some surgeons have reported good results without interposing any spacer between the retractors and the tarsus, ${ }^{5}$ others claim that a piece of sclera or cartilage is necessary. ${ }^{1,6,8}$ Tarsal flaps can be created to work as spacers. ${ }^{14}$ A Frost suture is often essential after operation on the lower lid. If there is lid laxity, an eyelid tightening procedure aids in raising the lower lid. ${ }^{8}$

Most patients are satisfied with correction of the upper eyelids and surgery on the lower lids is less often needed. In my experience, mere recessions have only been moderately effective and better correction has been achieved with tarsal flaps sutured to the edge of the retractors.

Since the retractors are easily accessible after a conjunctival incision, this is the procedure used by most surgeons. If a blepharoplasty is performed at the same time a skin incision is preferable.

\section{Complications of surgery}

The most frequent complications are listed in Table VIII. Undercorrection or overcorrec-

Table VIII Complications after surgery for eyelid retraction

\footnotetext{
Undercorrection

Overcorrection

Oblique and/or flat eyelid contour

Abnormal position of lid crease

Persistent eyelid oedema
}

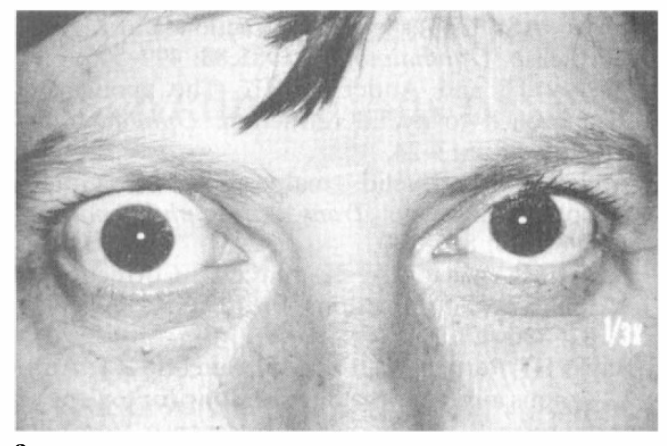

$\mathbf{a}$

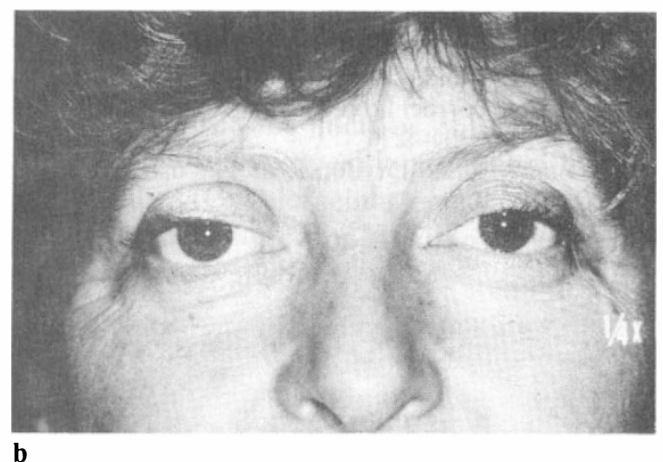

Fig. 3. Patient had bilateral operation for upper eyelid retraction, more marked on her right side [(a) before and (b) after surgery]. As a result of the operations, the upper eyelid crease is raised.

tion is best avoided by careful monitoring of the eyelid position during the operation but if this does occur, a reoperation is necessary with further recession or aponeurosis shortening. An oblique eyelid contour may follow if the lateral horn is not cut or a flat contour if the recession is too large in the central lid. These conditions may also require reoperation. A raised lid crease is not uncommon (Fig. 3), and may necessitate an adjustment by a skin incision. Persistent oedema has been reported in cases of homologous grafting. ${ }^{1.5 .6}$

\section{References}

'Chalfin J and Putterman AM: Müller's muscle excision and levator recession in retracted upper lid. Arch Ophthalmol 1979, 97:1487-91.

2Dixon R: The surgical management of thyroidrelated upper eyelid retraction. Ophthalmology 1982, 89: 52-7.

${ }^{3}$ Grove AS: Eyelid retraction treated by levator marginal myotomy. Ophthalmology 1980, 87: 101318. 
${ }^{4}$ Grove AS: Upper eyelid retraction and Graves' disease. Ophthalmology 1981,88: 499-506.

${ }^{5}$ Harvey JT and Anderson RL: The aponeurotic approach to eyelid retraction. Ophthalmology 1981, 88: 513-24.

${ }^{6}$ Waller RR: Eyelid malposition in Graves' ophthalmopathy. Trans Am Ophthalmol Soc 1982, 80: 855-930.

${ }^{7}$ Baylis HI, Cies WA, Kamin DF: Correction of upper eyelid retraction. Am J Ophthalmol 1976, 82: 790-4.

${ }^{8}$ Baylis HI, Perman KI, Fett DR, Sutcliffe RT: Autogenous auricular cartilage grafting for lower eyelid retraction. Ophthal Plast Reconstr Surg 1985, 1: 23-7.

${ }^{9}$ Callahan A: Levator recession. Arch Ophthalmol 1965, 73: 800-2.

${ }^{10}$ Crawford JS and Easterbrook M: The use of bank sclera to correct lid retraction. Can J Ophthalmol 1976, 11: 304-8.

"Doxanas MT and Dryden RM: The use of sclera in the treatment of dysthyroid eyelid retraction. Ophthalmology 1981, 88: 887-94.

12 Flanagan JC: Eye bank sclera in oculoplastic surgery. Ophthal Surg 1974, 5: 45-63.

${ }^{13}$ Henderson JW: Relief of eyelid retraction. Arch Ophthalmol 1965, 74: 205-16.

${ }^{14}$ Kohn R: Treatment of eyelid retraction with two pedicle tarsal rotation flaps. Am J Ophthalmol 1983, 95: 539-44.

${ }^{15}$ Putterman AM, Urist M: Surgical treatment of upper eyelid retraction. Arch Ophthalmol 1972, 87: 401-5.

16 Putterman AM and Fett DR: Müller's muscle in the treatment of upper eyelid retraction: A 12-year study. Ophthal Surg 1986, 17: 361-7.

${ }^{17}$ Hedin A: Is scleral transplantation necessary in the treatment of upper eyelid retraction? Orbit 1985 , 4: 115-20.

${ }^{18}$ Hawes MJ and Dortzbach RK: The microscopic anatomy of the lower eyelid retractors. Arch Ophthalmol 1982, 100: 1313-18. 